\title{
Analytic methods for the Percus-Yevick hard sphere correlation functions
}

\author{
D. Henderson* \\ Department of Chemistry and Biochemistry, Brigham Young University, Provo UT 84602
}

Received May 18, 2009, in final form June 9, 2009

The Percus-Yevick theory for hard spheres provides simple accurate expressions for the correlation functions that have proven exceptionally useful. A summary of the author's lecture notes concerning three methods of obtaining these functions are presented. These notes are original only in part. However, they contain some helpful steps and simplifications. The purpose of this paper is to make these notes more widely available.

Key words: Percus-Yevick, hard spheres, correlation functions, integral transforms, analytic results

PACS: 02.30.Qy, 02.30.Rz, 05.20.Jj, 05.70.Ce, 64.30.+t

\section{Introduction}

The hard sphere (HS) fluid is a simple representative fluid that, through perturbation theory [1, $2]$, is the basis of a simple and successsful theory of liquids. Hard spheres interact through the HS potential

$$
u(R)= \begin{cases}\infty, & R<d \\ 0, & R>d\end{cases}
$$

where $R$ is the separation of a pair of spheres and $d$ is their diameter. The reason why the HS fluid is so useful is that accurate analytic expressions can be obtained from the Percus-Yevick (PY) theory [3]. Indeed, perturbation theory could have been developed and used decades earlier had the PY expressions been available. The PY theory and the mean spherical approximation (MSA) [4] are identical for the HS fluid. Since the MSA is more widely used, perhaps the PY results for hard spheres should be called the MSA results. However, the name PY is conventionally associated with these results so the conventional nomenclature will be used.

The PY theory produces analytic results for the HS thermodynamics, direct correlation function $(\mathrm{DCF}), c(R)$, and the Laplace transform of the radial distribution function (RDF), $g(R)$. The DCF and RDF are related through the Ornstein-Zernike (OZ) equation,

$$
h\left(R_{12}\right)=c\left(R_{12}\right)+\rho \int h\left(R_{13}\right) c\left(R_{23}\right) \mathrm{d} \mathbf{r}_{3},
$$

where $R_{i j}=\left|\mathbf{r}_{i}-\mathbf{r}_{j}\right|$ is the separation of a pair of spheres located at $\mathbf{r}_{i}$ and $\mathbf{r}_{j}$ and $\rho=N / V$ is the number of spheres divided by the volume. The function, $h(R)=g(R)-1$ is called the total correlation function (TCF). Because of the discontinuity in the HS potential, it is useful to define the background function or cavity function, $y(R)$, by $g(R)=e(R) y(R)$, where $e(R)=\exp [-\beta u(R)]$ and $\beta=1 / k_{\mathrm{B}} T$ with $k_{\mathrm{B}}$ and $T$ being the Boltzmann constant and temperature, respectively. The background function is useful because it is continuous since the discontinuities of $g(R)$ and $c(R)$ arise solely from $e(R)$, which is zero for $R<d$ and one for $R>d$. The integral in the OZ equation is a convolution integral.

The OZ equation is a definition of the DCF and does not, by itself, yield a theory. The PY theory for the HS fluid is obtained by combining the OZ equation together with both the exact

*E-mail: doug@chem.byu.edu 
result for the HS fluid,

$$
h(R)=-1, \quad R<d,
$$

and the approximation,

$$
c(R)=0, \quad R>d .
$$

The DCF is, in a sense, an inverse of the TCF. To see this, the Fourier transform, $\tilde{f}(k)$, of a spherically symmetric function, $f(R)$, is defined by

$$
k \tilde{f}(k)=4 \pi \int_{0}^{\infty} R \sin (k R) f(R) \mathrm{d} R
$$

with the inverse transform given by

$$
R f(R)=\frac{1}{2 \pi^{2}} \int_{0}^{\infty} k \sin (k R) \tilde{f}(k) \mathrm{d} k .
$$

Note that $\tilde{f}(k)=\tilde{f}(-k)$. Making use of the fact that the Fourier transform of a convolution integral is the product of the Fourier transforms of the constituent functions, the OZ equation, when transformed, can be written as

$$
1+\rho \tilde{h}(k)=[1-\rho \tilde{c}(k)]^{-1} .
$$

The thermodynamic functions can be calculated from the correlation functions using the equations,

$$
\frac{p V}{N k_{\mathrm{B}} T}=1+\frac{2 \pi}{3} \rho d^{3} y(d)
$$

and

$$
\frac{1}{k_{\mathrm{B}} T} \frac{\partial p}{\partial \rho}=1-4 \pi \rho \int_{0}^{\infty} R^{2} c(R) \mathrm{d} R
$$

where $p$ is the pressure. Because $c(R)$ and $h(R)$ are related through the OZ equation, equation (9) can also be written as

$$
k_{\mathrm{B}} T \frac{\partial \rho}{\partial p}=1+4 \pi \rho \int_{0}^{\infty} R^{2} h(R) \mathrm{d} R
$$

It is to be noted that the integrals in equations (9) and (10) are $\tilde{c}(0)$ and $\tilde{h}(0)$, respectively. Equation (8) is exact for a HS fluid; equations (9) and (10) are exact for any simple liquid. Of course, in the PY theory for a HS fluid, the upper limit for the integration in equation (9) is actually $d$. Equation (8) is often called the pressure (p) equation or virial equation. Equation (9) [and equation (10)] is often called the compressiblity (c) equation. Note that, for the HS fluid, $y(d)=g\left(d^{+}\right)$is valid, exactly. In the PY theory for hard spheres, $c\left(d^{-}\right)=-y(d)$. More generally, $c(R)=-y(R)$, for $R<d$, in the PY theory for hard spheres.

Another equation that is useful is

$$
y(0)=-c(0)=1-4 \pi \rho \int_{0}^{d} R^{2} c(R) \mathrm{d} R=\beta\left(\frac{\partial p}{\partial \rho}\right)_{(c)} .
$$

This equation, which we may call the PY zero separation equation, is valid only for the PY theory for hard spheres. It is obtained from the $\mathrm{OZ}$ equation, equation (2), in the limit $R_{12}=0$, together with equations (3) and (4). The first term after the left equal sign is just $h(0)=-1$. The following term is the integral in the $\mathrm{OZ}$ equation(in the limit $R_{12}=0$ ), using $h(R) c(R)=-c(R)$ for $R<d$ and zero for $R>d$. It is interesting to note that there is an exact zero separation theorem, $y(0)=\exp (\beta \Delta \mu)$, where $\Delta \mu$ is the chemical potential of the hard sphere fluid in excess of that of an ideal gas. This exact theorem follows from the fact that for hard spheres at zero separation, one hard sphere has disappeared inside the other hard sphere and, in fact, one hard sphere has been removed from the system. The exact theorm follows because the chemical potential is the energy needed or gained to add or remove a particle from the system. The PY/HS theory does not even come 
close to satisfying the exact zero separation theorem. The combination of equation (11) and the exact theorem would give a terrible result for the chemical potential. None-the-less, equation (11) is useful for obtaining an expression for the PY/HS $c(R)$.

Of course, if exact results were available, the $(\mathrm{p})$ and $(\mathrm{c})$ equations would be consistent. However, because the PY theory is approximate, the pressure that results from the (p) and (c) equations will be inconsistent and slightly different. The thermodynamic functions used in the zero separation equation (11) are identical to the (c) equation results.

As has been stated, the PY theory is important for the HS fluid because it yields analytic expressions for $c(R)$ and explicit results for $g(R)$ and $y(R)$ that are quite accurate. Three methods for obtaining these functions will be considered. The results presented here come from the author's lecture notes. One of the methods (the first) given here is probably original. However, when the methods are not original, the steps and simplifications that are given have proven useful. For example, some of these notes were found to be valuable by Gray and Gubbins and appear in an appendix in their well-known monograph [5]. The author has decided to publish these lecture notes, as a review, so that they might be more widely available.

\section{Simple method}

Even before the correlation functions for hard spheres were obtained, it was realized that the virial coefficients for this fluid were particularily simple. The virial series is a power series in $\rho$ for the pressure,

$$
\frac{p V}{N k_{\mathrm{B}} T}=1+\sum_{n=2}^{\infty} B_{n} \rho^{n-1} .
$$

The coefficients, $B_{n}$, are called the virial coeffients. Using the PY theory, Hutchinson and Rushbrooke [6] obtained first seven $B_{n}$, as given by the $\mathrm{p}$ and c equations. Their results are given by the formulae,

$$
B_{n}^{p}=\frac{2(3 n-4)}{4^{n-1}} b^{n-1}
$$

and

$$
B_{n}^{c}=\frac{1+\frac{3}{2} n(n-1)}{4^{n-1}} b^{n-1},
$$

where $b=2 \pi d^{3} / 3$. The p equation virial coefficients are somewhat too small but are reasonably good. The c equation virial coefficients are even more accurate. They are slightly too large.

Assuming these expressions to be generally valid, the power series can be summed to yield

$$
\left(\frac{p V}{N k_{\mathrm{B}} T}\right)_{(p)}=\frac{1+2 \eta+3 \eta^{2}}{(1-\eta)^{2}}
$$

and

$$
\left(\frac{p V}{N k_{\mathrm{B}} T}\right)_{(c)}=\frac{1+\eta+\eta^{2}}{(1-\eta)^{3}}
$$

where $\eta=\pi \rho d^{3} / 6$ is the volume occupied by the spheres divided by the volume available and is called the volume fraction of the fluid. It is quite possible that equations (13) and (14) could be shown by induction to be correct to all orders of $n$. This has never been done. However, the other methods considered in subsequent sections show that equations (15) and (16) are valid so there is not much incentive for an induction proof. We will accept the above results in this section.

As has been observed for the virial coefficients, the (c) equation result for $p V / N k_{\mathrm{B}} T$ is slightly too large whereas the (p) equation result for this quantity is a little more in error and is somewhat small. This suggests that a linear combination of the $(p)$ and (c) equation results will give a good description for the hard sphere pressure. Indeed, the well-known Carnahan-Starling formula, that is given by $p V / N k_{\mathrm{B}} T=\left(p V / N k_{\mathrm{B}} T\right)_{(p)} / 3+2\left(p V / N k_{\mathrm{B}} T\right)_{(c)} / 3$, gives good agreement with simulation results for the hard sphere equation of state. 
From equation (15) we can deduce that the result of the PY theory for the contact value of the background function is

$$
y(d)=\frac{1+\eta / 2}{(1-\eta)^{2}}
$$

Remember that $g\left(d^{+}\right)=y(d)$ and $c\left(d^{-}\right)=-y(d)$ for PY/HS. Another result of interest is the compressiblity, as given by the compressiblity equation,

$$
\left(\frac{1}{k_{\mathrm{B}} T} \frac{\partial p}{\partial \rho}\right)_{(c)}=\frac{(1+2 \eta)^{2}}{(1-\eta)^{4}} .
$$

It is of interest to note that this is the square of a simple expression. We will return to this point in the next section.

Also, it was found that at least through $\rho^{2}$, the coefficients in the density expansion of the PY DCF is a simple polynomial. Specifically,

$$
c(R)=c_{0}+c_{1} \frac{R}{d}+c_{3}\left(\frac{R}{d}\right)^{3} .
$$

for $R<d$ and zero for $R>d$. Presumably, it could be proven by induction that this expression is valid to all orders in the density expansion. This has not been done. There is little incentive to do this as the general validity of this expression has been established by general means that we shall discuss in the following sections.

There are three unknowns in equation (19). Fortunately, we have three conditions to employ. We know $c(0)$ and, hence,

$$
c_{0}=-\frac{(1+2 \eta)^{2}}{(1-\eta)^{4}} .
$$

Further, we know $c\left(d^{-}\right)$and the integral of $c(R)$. Hence,

$$
c_{1}=6 \eta \frac{(1+\eta / 2)^{2}}{(1-\eta)^{4}}
$$

and

$$
c_{3}=\frac{\eta}{2} c_{0}
$$

Thus, the DCF is completely determined.

It is worth noting that the pressure may be integrated to give the Helmholtz function, yielding

$$
\left(\frac{A-A_{0}}{N k_{\mathrm{B}} T}\right)_{(p)}=2 \ln (1-\eta)+6 \frac{\eta}{1-\eta}
$$

and

$$
\left(\frac{A-A_{0}}{N k_{\mathrm{B}} T}\right)_{(c)}=\frac{3 \eta(1-\eta / 2)}{(1-\eta)^{2}}-\ln (1-\eta),
$$

where $A_{0}$ is the Helmholtz function of an ideal gas.

\section{Fourier transform method}

Baxter [9] has published an attractive method, based on the Fourier transform, for determining the correlation functions. This appeared after the Laplace transform method that will be presented in the next section. It is generally regarded as a superior method. The author does not share this view. In his view, the Laplace transform method is more powerful and yields useful results that are either not available from the Fourier transform method or have not been obtained by this method. The basis of the author's statement is that if the Laplace transform is known, the Fourier 
transform may be determined. However, the reverse is not true. Nonetheless, Baxter's method is pedagogically attractive.

The compressiblity is positive or zero and cannot be negative, in a thermodynamically stable system. Thus, it is plausible that the compressiblity should be a simple square, as is the case with equation (18). More generally, using the fact that the DCF vanishes for $R>d$, Baxter has shown by a Wiener-Hopf factorization that

$$
1-\rho c \tilde{(k)}=\tilde{Q}(k)^{2}=\tilde{Q}(k) \tilde{Q}(-k)
$$

The aforementioned observation that the compressiblity is a simple square is seen from equation (25) with $k=0$. Additionally, this factorization permits the OZ equation, which involves a three-dimensional convolution, to be written as two equations that each involve only one dimensional convolutions,

$$
R c(R)=-Q^{\prime}(R)+2 \pi \rho \int_{0}^{d} Q^{\prime}(S) Q(S-R) \mathrm{d} S
$$

and

$$
R h(R)=-Q^{\prime}(R)+2 \pi \rho \int_{0}^{d}(R-S) h(|R-S|) Q(S) \mathrm{d} S,
$$

where $Q(R)$ has the Fourier transform, $\tilde{Q}(k)$, defined by

$$
\tilde{Q}(k)=1-2 \pi \rho \int_{0}^{d} \exp (\mathrm{i} k R) Q(R) \mathrm{d} R .
$$

The function $Q(R)$ is zero for $R>d$. The compressibility can be written in the form

$$
\beta \frac{\partial p}{\partial \rho}=\tilde{Q}(0)^{2}
$$

Considering equation (27) for $R<d$ yields

$$
Q^{\prime}(S)=a S+b
$$

with

$$
a^{2}=c_{0}
$$

and

$$
b=-\frac{3 \eta}{2(1-\eta)^{2}}
$$

Thus,

$$
Q(S)=\frac{1}{2} a\left(S^{2}-d^{2}\right)+b(S-d)
$$

Equations (19)-(22) now follow from equation (26). This provides a justification for the assumption in the preceeding section that the HS/PY DCF is a simple polynomial and the form for the compressibility equation and pressure equation thermodynamics that was assumed in the previous section.

It is worth noting that Baxter has shown that, in the PY theory, the pressure calculated from

$$
\begin{aligned}
\left(\frac{p V}{N k_{\mathrm{B}} T}\right)_{(c)}= & 1-4 \pi \rho \int_{0}^{\infty} R^{2} c(R) \mathrm{d} R+2 \pi \rho \int_{0}^{\infty} R^{2} e(R)[e(R)-1] y^{2}(R) \mathrm{d} R \\
& +\frac{1}{2 \pi^{2} \rho} \int_{0}^{\infty} k^{2}[\rho \tilde{c}(k)+\ln \{1-\rho \tilde{c}(k)\}] \mathrm{d} k
\end{aligned}
$$

gives the same pressure as that obtained from integrating $\partial p / \partial \rho$ from the compressibility equation pressure. Equation (34) is valid for a general (simple) fluid. The derivation of equation (34) is not straightforward but it is quite easy to verify equation (34) by differentiating this equation, using 
the PY approximation, and obtain equation (9). This equation has the advantage that the solution of the PY equation need not be known for a sequence of states. This is no advantage for the HS fluid, where a general analytic solution that is valid for all states is available. However, for systems for which a numerical result must be obtained for each state, this is a real advantage especially if there is a thermodynamically unstable region that must be crossed. Regretably, a further result yielding the Helmholtz function is not available.

\section{Laplace transform method}

Thiele [7] and Wertheim [8] have obtained the PY/HS thermodynamics and correlation functions using Laplace transform techniques. The method involves more algebra than the Fourier transform method just discussed and has lead to what I feel is the incorrect conclusion that the Fourier transform is to be preferred. My opinion is based on the fact that the Laplace transform method yields both $c(R)$ and $g(R)$. The expression for $c(R)$ is the analytic result that has been obtained by the previous methods. In addition, an analytic expression for $G(s)$, the Laplace transform of $g(R)$, is obtained. The inversion of $G(s)$ is not analytic but useful explicit results for $g(R)$ can be obtained.

The Laplace transform $F(s)$ of a function $f(R)$ may, in the present context, be defined by

$$
F(s)=\int_{0}^{\infty} x f(x) \exp (-s x) \mathrm{d} x
$$

where $x=R / d$. Thus,

$$
C(s)=\int_{0}^{\infty} x c(x) \exp (-s x) \mathrm{d} x-\int_{1}^{\infty} x c(x) \exp (-s x) \mathrm{d} x .
$$

Wertheim shows quite rigorously that the PY/HS DCF is given by equations (19)-(22). However, let us keep things simple here by assuming these results. Now, let us evaluate the first integral in equation (36) by assuming that equation (19), with equations (20)-(22), is valid for all $x$.

Thus,

$$
C(s)=\frac{c_{0}}{s^{2}}+\frac{c_{1}}{s^{3}}+\frac{c_{3}}{s^{5}}-\exp (-s) \int_{0}^{\infty}(t+1) c(t+1) \exp (-s t) \mathrm{d} t .
$$

The second and third terms in equation (37) can be combined. Hence,

$$
\frac{c_{1}}{s^{3}}+\frac{c_{3}}{s^{5}}=-12 \eta \frac{L(s) L(-s)}{(1-\eta)^{4} s^{5}}
$$

where

$$
L(s)=(1+2 \eta)+(1+\eta / 2) s .
$$

Furthermore, the last term in equation (37) can be written in a form that involves $L(s)$. After a little algebra,

$$
C(s)=\frac{c_{0}}{s^{2}}-12 \eta \frac{L(s) L(-s)}{(1-\eta)^{4} s^{5}}-\exp (-s) \frac{L(s) S(-s)}{(1-\eta)^{4} s^{5}}
$$

where

$$
S(s)=-12 \eta(1+2 \eta)+18 \eta^{2} s+6 \eta(1-\eta) s^{2}+(1-\eta)^{2} s^{3} .
$$

The polynomials $L(s)$ and $S(s)$ may be called Wertheim polynomials.

We are now in a position to obtain the Laplace transform of $g(R)$. The OZ equation may be written in the form

$$
g\left(R_{12}\right)-c\left(R_{12}\right)=\frac{(1+2 \eta)^{2}}{(1-\eta)^{4}}+\rho \int g\left(R_{13}\right) c\left(R_{23}\right) \mathrm{d} \mathbf{r}_{3}
$$


where equations (9) and (18) have been used. After a little algebra,

$$
G(s)-C(s)=-\frac{c_{0}}{s^{2}}+\frac{12 \eta}{s} G(s)[C(-s)-C(s)] .
$$

Solving for $G(s)$ gives

$$
G(s)=\frac{-c_{0} / s^{2}+C(s)}{1+12 \eta[C(s)+C(-s)] / s} .
$$

Using a little algebra and the identity,

$$
(12 \eta)^{2} L(s) L(-s)-(1-\eta)^{4} s^{6}=S(-s) S(s),
$$

leads to the desired expression for $G(s)$

$$
G(s)=\frac{s L(s)}{12 \eta L(s)+\exp (s) S(s)} .
$$

This result for $G(s)$ can be inverted explicitly $[10,11]$ to yield results for $g(R)$ for $R \leqslant 5 d$. Also, this expression can be used to obtain a number of valuable results. For example, the contact value, $g\left(d^{+}\right)$, can be obtained from the limit of $s G(s)$ at large $s$. The result is equation (17). Also, we can expand $G(s)$ in powers of $s$.

$$
\frac{s L(s)}{12 \eta L(s)+\exp (s) S(s)}=\frac{1}{s^{2}}-\frac{10-2 \eta+\eta^{2}}{20(1+2 \eta)}+\frac{(4-\eta)(2+\eta)}{24(1+2 \eta)^{2}} s^{2}+\cdots .
$$

Recognizing that

$$
G(s)=\frac{1}{s^{2}}+\int_{0}^{\infty} x h(x) \exp (-s x) \mathrm{d} x
$$

we obtain, by equating coefficients of like powers of $s$,

$$
\int_{0}^{\infty} x h(x) \mathrm{d} x=-\frac{10-2 \eta+\eta^{2}}{20(1+2 \eta)}
$$

and

$$
\int_{0}^{\infty} x^{2} h(x) \mathrm{d} x=-\frac{(4-\eta)\left(2+\eta^{2}\right)}{24(1+2 \eta)^{2}} .
$$

Equation (50) yields the PY/HS compressiblity that has been obtained previously. This process can be used to obtain $\int_{0}^{\infty} x^{n} h(x) \mathrm{d} x$ for any $n$. Since the error in the PY $g(R)$ is mostly in the region of $g(R)$ near $d$, these integrals become more accurate as $n$ increases.

Alternatively, we can use equation (48) and obtain

$$
\int_{0}^{\infty} x^{n+1} h(x) \mathrm{d} x=\lim _{s \rightarrow 0}\left[(-1)^{n} \frac{d^{n} G(s)}{d s^{n}}-\frac{(n+1) !}{s^{n+2}}\right],
$$

for $n \geqslant 1$. Integrals involving $x^{-n}$ can be evaluated from

$$
\int_{1}^{\infty} x^{-n} g(x) \mathrm{d} x=\frac{1}{n !} \int_{0}^{\infty} s^{n} G(s) \mathrm{d} s,
$$

for $n>1$. For these latter integrals, the value of the integral will become less accurate as $n$ increases. In the case where $n$ is 6 and 12, these integrals can be used in a perturbation theory for the Lennard-Jones potential.

This leaves the two integrals

$$
\int_{0}^{\infty} h(x) \mathrm{d} x=\int_{0}^{\infty}\left[G(s)-\frac{1}{s^{2}}\right] \mathrm{d} s
$$


and

$$
\int_{1}^{\infty} \frac{h(x)}{x} \mathrm{~d} x=\int_{0}^{\infty} s\left[G(s)-\frac{s+1}{s^{2}} \exp (-s)\right] \mathrm{d} s,
$$

that are easily obtained from

$$
\int_{0}^{\infty} x h(x) \exp (-s x) \mathrm{d} x=G(s)-\frac{1}{s^{2}}
$$

and

$$
\int_{1}^{\infty} \frac{h(x)}{x} \mathrm{~d} x=G(s)-\frac{s+1}{s^{2}} \exp (-s) .
$$

Lebowitz [12] has applied the Laplace transform method to obtain corresponding PY expressions for the direct correlation functions and Laplace transforms of the radial distribution functions and the resulting thermodynamic functions for a mixture of hard spheres with additive diameters.

\section{Summary}

Results, drawn from the author's lecture notes, for the solution of the PY equation for a hard sphere fluid that have proven useful and that have appeared, in part, in the monograph of Gray and Gubbins [5] are presented so as to make them more widely available. To the author's knowledge, only section 2 is original. The remainder of the article is a mini review, with simplifications, that it is hoped will be of interest.

\section{Acknowledgement}

Jean-Pierre Hansen read a draft of this paper and made valuable suggestions for which the author is grateful. However, any errors in this paper are the responsiblity of the author.

\section{References}

1. Barker J.A., Henderson D., J. Chem. Phys., 1967, 47, 2856, 4714; Rev. Mod. Phys., 1976, 48, 587.

2. Hansen J.-P., McDonald I.R. Theory of Simple Liquids, 3rd. Edition. Academic Press, London, UK, 2006.

3. Percus J.K., Yevick G.J., Phys. Rev., 1958, 110, 1.

4. Lebowitz J.L., Percus J.K., Phys. Rev., 1966, 144, 251.

5. Gray C.G., Gubbins K.E. Theory of Molecular Fluids. Volume 1: Fundamentals. Clarendon Press, Oxford, UK, 1984, Appendix 5A.

6. Hutchinson P., Rushbrooke G.S., Physica, 1963, 29, 675.

7. Thiele E., J. Chem. Phys., 1963, 39, 474.

8. Wertheim M., Phys. Rev. Lett., 1963, 10, 321 (1963); J. Math. Phys., 1964, 5, 643.

9. Baxter R.J., Aust. J. Phys., 1968, 21, 563.

10. Smith W.R., Henderson D., Mol. Phys., 1970, 19, 411.

11. Smith W.R., Henderson D., Leonard P.J., Barker J.A., Grundke E.W., Mol. Phys., 2007, 106, 3.

12. Lebowitz J.L., Phys. Rev. A, 1964, 133, 895. 


\title{
Аналітичні методи для кореляційних функцій твердих сфер у наближенні Перкуса-Євіка
}

\author{
Д.Гендерсон \\ Факультет хімії і біології, Університет Брайхем Янг, Прово, США \\ Отримано 18 травня 2009 р., в остаточному вигляді - 9 червня 2009 р. \\ Теорія Перкуса-Євіка для твердих сфер дає можливість отримати дуже корисні прості точні вирази \\ для кореляційних функцій. Тут представлено підсумкові нотатки лекцій, що стосуються трьох ме- \\ тодів отримання цих функцій. Представлені нотатки $є$ тільки частково оригінальними. Проте, вони \\ містять корисні кроки і спрощення. Метою цієї статті $є$ зробити ці нотатки більш доступними широ- \\ кому загалу.
}

Ключові слова: Перкус-Євік, тверді сфери, кореляційні функції, інтегральні перетворення, аналітичні результати

PACS: $02.30 . Q y, 02.30 . R z, 05.20 . J j, 05.70 . C e, 64.30 .+t$ 
